\title{
To investigate and analyze the current using situation of nasointestinal tube in emergency department of 126 general hospitals in Guangdong province
}

\author{
Huang Xuanjie ${ }^{1 *}$,Deng Lisi ${ }^{2 *}$, Nie Fali ${ }^{3}$ Zhang Zhiming ${ }^{4}$, Zhou Hao ${ }^{5 \#}$ \\ ${ }^{1}$ Emergency Department, the Seventh Affiliated Hospital, Sun Yat-sen University, Shen Zhen,518107; Huang Xuan-jie, \\ Email E-mail: 190829610@qq.com,https://orcid.org/0000-0002-8811-7937 \\ ${ }^{2}$ Huadu District People's Hospital,Guangzhou, 510400,Deng Li-si, Email: 103909986@qq.com,https://orcid.org/0000- \\ 0003-1116-5698 \\ ${ }^{3}$ Shenzhen Hospital of Southern Medical University, Shen Zhen, 518110 \\ ${ }^{4}$ Dongguan Donghua Hospital, Dongguan, 523015 \\ ${ }^{5}$ Department of Emergency Guangzhou First People's Hospital, School of Medicine, South China University of Technology, \\ Guangzhou, 510000 \\ * Huang Xuan-jie and Deng Li-si are co-first authors. \#Zhou Hao is the corresponding author.
}

Article History Received 22 March 2020 Accepted 22 April 2020 Published 30 June 2020

Cite this Article Huang Xuanjie,Deng Lisi, Nie Fali, Zhang Zhiming, Zhou Hao To investigate and analyze the current using situation of nasointestinal tube in emergency department of 126 general hospitals in Guangdong province [J].Medical Research, 2020.2(2):5-11, http://dx.doi.org/10.6913/MRHK.202006_2(2).0 003

Copyright C 2020 Creative Publishing Co., Limited. All rights reserved. mrhk26640333@gmail.com.

\section{ABSTRACT}

Objective To analyze the status and cognition of nasointestinal tube in emergency department of 126 general hospitals in Guangdong province.

Methods The self-designed general information questionnaire for knowledge, belief and behavior was used to investigate 602 emergency nurses of 126 general hospitals.

Results 1. The cognitive score of emergency nurse on nasointestinal tube showed: The score of knowledge was $(8.24+5.02)$, the qualified rate was $52.2 \%$. the score of belief was $(23.82+5.03)$, and the score of behavior was $(29.77+18.71)$. Multiple linear regression results showed that The emergency nurses' cognition of nasointestinal tube was influenced by Working age, professional ranks, level, educational background and hospital grade. $\quad(\mathrm{P}<0.01)$ 2.Correlation analysis showed that the scores of attitude and behavior of nurses with different educational background, professional ranks and working age were significantly different.

Conclusion Emergency nurse have a low cognition of nasointestinal tube in Guangdong Province. It is necessary to study the knowledge and technology of nasointestinal tube, make standardized operating guideline and procedure, to further improve the emergency nurses'essary to study the knowledge and technology of nasointestinal tube nificantly different.

Key words Nasointestinal tube; Emergency department; Nurses; Cognition; Questionnaires

\section{INTRODUCTION}

According to The Clinical Resource Efficiency Team, the naso-intestinal tube is mainly used for enteral 
Huang Xuan-jie et al. To investigate and analyze the current using situation of nasointestinal tube in emergency department Medical Research ISSN 2664-0333 eISSN 2664-0341 Volume 2 Issue 2 http://dx.doi.org/10.6913/MRHK.202006_2(2).0003

nutrition therapy. It can be any nasal feeding tube that passes through the esophagus and pylorus and is usually named after the end location of the tube. For instance, if the end of the tube is in the duodenum, it is called a naso-duodenal tube; and if the end of the tube is in the jejunum, it is called a naso-jejunal tube ${ }^{[1]}$. The nutrition guidelines of the American Society of Critical Care Medicine (SCCM) and the American Society for Parenteral and Enteral Nutrition (ASPEN) ${ }^{[7]}$ recommend using naso-intestinal tube feeding when a patient is intolerant of naso-gastric tubes for enteral nutrition but convenient for naso-intestinal tube implantation. A domestic study of elderly patients $(83 \sim 103$ years old) found that the utilization of naso-intestinal tubes for enteral nutrition can reduce the incidence of vomiting, reflux, aspiration, diarrhea, abdominal distension, and pulmonary infections ${ }^{[5]}$.

With the rapid development of medicine, the regional distribution of emergency departments has covered the rescue area, observation area, emergency ward, and emergency intensive care unit (EICU). Elderly patients in emergency observation generally suffer from malnutrition ${ }^{[10]}$, and the number of patients using nasointestinal tubes is increasing year by year. Therefore, emergency nurses are required to master the knowledge and skills related to naso-intestinal tubes. To prospectively understand emergency nurses' overall understandings of the naso-intestinal tube, this investigation was conducted and the current situation was analyzed. Particularly, this study investigated the status of the utilization of naso-intestinal tubes in the emergency department through three aspects including knowledge, belief, and practice, with the aim to provide a reference for the future application of naso-intestinal tubes in emergency departments.

\section{MATERIALS AND METHODS}

In October 2018, a total of 602 nurses in 126 emergency departments of general hospitals in Guangdong province in China were surveyed by the convenience sampling method. Inclusion criteria: (1) active and registered nurses; (2) at least 1-year working experience in the emergency department. Exclusion criteria: (1) training nurses; (2) those who are unable to participate in the survey due to sick leave, personal leave, or under training outside of the hospital at the time of the survey.

(1) The general information questionnaire was self-designed by the researchers. The questionnaire covered the information related to gender, working years, education, professional title, level, hospital grade, average daily emergency visit volume, common emergency diseases, the age range of emergency patients, and the emergency area.

(2) The questionnaire on emergency nurses' knowledge, belief, and practice about naso-intestinal tube was designed by the researchers based on relevant literature ${ }^{[2-3]}$. This self-designed questionnaire entitled " $A$ Survey on the Current Situation of the Utilization of Naso-intestinal Tube in the Emergency Departments among Hospitals in Guangdong Province" was gradually revised and formed after the discussion by the research team and inquiries from the experts such as the Chief Nurse Shuxia Li. The questionnaire included three dimensions, namely the naso-intestinal tube knowledge dimension (9 items), belief dimension (6 items), and practice dimension (10 items). The knowledge dimension included the indications of the naso-intestinal tube, the scope of use, the preferred route of patient nutritional intake (e.g., which feeding route should be chosen for patients with enteral nutrition less than 6 weeks and with a high risk of reflux and aspiration), the position of enteral nutrition, the appropriate temperature of the nasogastric nutrient solution, the gold standard for confirming the position of the naso-intestinal tube, the amount of tubing flushing, and the preferred infusion method, etc. Single-choice and multiple-choice questions were used. Single-choice questions were 1 point for correct answers and no points for wrong answers. Multiple-choice questions were 1 point for each right answer. The total score was 16 points, and the correct rate with more than $60 \%$ was considered as qualified or passing. The high score was corresponding to the high the awareness of early activities of the emergency nurses. Belief dimensions included the importance of naso-intestinal tube technology in emergency departments, the 
Huang Xuan-jie et al. To investigate and analyze the current using situation of nasointestinal tube in emergency department Medical Research ISSN 2664-0333 eISSN 2664-0341 Volume 2 Issue 2 http://dx.doi.org/10.6913/MRHK.202006_2(2).0003

importance of naso-intestinal tube nutrition support for critically ill patients in emergency departments, the advantages of naso-intestinal tube nutrition support, and approval to implementing naso-intestinal tube nutrition support to protect patients, etc. The Likert 5-level evaluation method was used. "Strongly agree" was 5 points, "Agree" was 4 points, "General" was 3 points, "Disagree" was 2 points, "Strongly disagree" was 1 point. The score range was between 6 to 30 points, and the score was used to evaluate the belief of emergency nurses on the naso-intestinal tube. Practice dimension refers to the situation in which emergency nurses perform naso-intestinal tube-related care for patients. Again, Likert 5-level scoring method was used: "always" was 5 points, "often" was 4 points, "sometimes" was 3 points, "occasionally" was 2 points, and "Never" was 1 point, and the score ranged from 10-50 points. The high score was corresponding to the practice performance of emergency nurses on naso-intestinal tube care. Experts such as the Chief Nurse Shuxia Li reviewed the questionnaire and 15 emergency nurses were selected for the pre-test. The Cronbach' $\alpha$ coefficient of the questionnaire was 0.979 .

The researchers obtained the consent and cooperation of deans of all hospitals and departments before the survey and determined the number of questionnaires to be issued and the survey time according to the specific conditions of each department. The researcher indicated the purpose of the survey on the first page of the questionnaire, and then filled in and submitted it after obtaining the informed consent of the research subjects. In this study, 621 questionnaires were collected and 19 were eliminated. Thus, 602 questionnaires were valid, with an effective rate of $96.94 \%$.

\section{STATISTICAL METHOD}

SPSS 24.0 software was used for statistical analysis. Emergency nurses, general information of the hospital, the scores of emergency nurses' knowledge, belief, and practice on the naso-intestinal tube were described in terms of frequency, percentage, and standard deviation (SD), etc. Multiple logistic regression analysis was used to compare the knowledge, belief, and practice scores of emergency nurses with different characteristics on the naso-intestinal tube. $\mathrm{P}<0.05$ indicated that the difference was statistically significant.

\section{RESULT}

The nurses in the emergency department were mainly distributed over 6 years of working experience, of which 418 (69.4\%) were from grade III level A hospitals, 62 (10.3\%) were from grade III hospitals, 97 (16.1\%) were from grade II level A hospitals, and 25 (4.2\%) were from grade II hospitals. The hierarchical distribution of these nurses was mainly N1, N2, and N3, and the majority of them had a college's or higher degree. See

Table 1 for details.

The daily emergency visit volume, common diseases, and regions of the nurses who participated in the survey (Table 2).

Emergency nurses' scores on belief dimension, practice dimension, and knowledge dimension of nasointestinal tube were $28.82 \pm 5.03,29.77 \pm 18.71$, and $8.24 \pm 5.02$, respectively. The knowledge, belief, and practice of emergency nurses on naso-intestinal tube were overall qualified. See Table 3 for details.

The total number of qualified emergency nurses on naso-intestinal tube knowledge was 314 (52.2\%). The top three passing questions regarding the knowledge of naso-intestinal tube are as followed. The correct rate was $86.98 \%$ on the question "which one do you think is the preferred way for patient's nutrition intake". The correct rate was $74.38 \%$ on the question "which feeding method should be selected for the patient with enteral nutrition $<6$ weeks and a high risk of reflux and aspiration". The correct rate was $67.36 \%$ on the question "what temperature is appropriate for the nasal feeding nutrient solution during nasal feeding". Moreover, the least three passing questions were "the preferred method of naso-intestinal infusion tube", "the scope of the use of naso-intestinal tube", and "indications of the naso-intestinal tube". The correct rates on these three questions were $26.45 \%, 27.6 \%$, and $29.9 \%$, respectively. 
There were $511(84.9 \%)$ emergency nurses passed with a total score of belief on naso-intestinal tubes. Among them, 383 (62\%) agreed with the statement "it is of great significance to carry out naso-intestinal tube technology in the emergency department"; 482 (77.9\%) agreed that "the implementation of naso-intestinal nutritional support protects the interests of patients"; and $512(82.8 \%)$ agreed with the statement "it is very important to master theoretical knowledge and skills related to naso-intestinal tubes".

Table 1. Basic information of nurses in emergency departments (\%)

\begin{tabular}{lll}
\hline \multirow{2}{*}{ Gender } & \multicolumn{1}{c}{ Items } & \multicolumn{1}{c}{ Number (\%) } \\
& Male & $109(18.1)$ \\
& Female & $493(81.9)$ \\
\hline \multirow{3}{*}{ Working years } & < 3 years & $115(19.1)$ \\
& 3 to 5 years & $114(18.9)$ \\
& 6-10 years & $160(26.6)$ \\
& More than 11 years & $213(35.4)$ \\
\hline \multirow{3}{*}{ Job title } & Nurse & $186(30.9)$ \\
& Nurse practitioner & $220(36.5)$ \\
& Nurse-in-charge & $166(27.6)$ \\
& Deputy director nurse and higher & $30(5.0)$ \\
\hline \multirow{5}{*}{ Education } & Technical secondary school & $28(4.7)$ \\
& College & $192(31.9)$ \\
& Bachelor & $378(62.8)$ \\
& Master or higher & $4(0.7)$ \\
\hline \multirow{3}{*}{ Hierarchy } & N1 & $197(32.7)$ \\
& N2 & $170(28.2)$ \\
& N3 & $168(27.9)$ \\
\hline
\end{tabular}

Table 2. Information of daily hospital visits and common diseases.

\begin{tabular}{lll}
\hline & Items & Number (\%) \\
\hline \multirow{3}{*}{ Average daily emergency } & $0-200$ & $131(21.8)$ \\
visit volume & $200-500$ & $340(56.5)$ \\
& $500-800$ & $92(15.3)$ \\
& $>800$ & $39(6.5)$ \\
\hline \multirow{2}{*}{ Common diseases } & Respiratory system diseases & $542(90.0)$ \\
& Nervous system diseases & $451(74.9)$ \\
& Circulatory system diseases & $453(75.2)$ \\
& Digestive system diseases & $459(76.2)$ \\
\hline \multirow{3}{*}{ Emergency regions } & The rescue area & $564(93.7)$ \\
& The observation area & $501(83.2)$ \\
& The emergency ward & $341(56.6)$ \\
& EICU & $263(43.7)$ \\
\hline
\end{tabular}

There were 381 (63.3\%) emergency nurses passed the naso-intestinal tube practice tests. The three items with the highest score were "properly fix feeding tubes (implementation rate: 62\%)", "enteral nutrient solution should be placed separately from intravenous medication (implementation rate: 62\%)", and "pay attention to patient's defecation condition (implementation rate: 58\%)", successively. The three items with the lowest 
Huang Xuan-jie et al. To investigate and analyze the current using situation of nasointestinal tube in emergency department Medical Research ISSN 2664-0333 eISSN 2664-0341 Volume 2 Issue 2 http://dx.doi.org/10.6913/MRHK.202006_2(2).0003

scores were successively "monitoring of gastric residues at regular intervals (implementation rate 42\%)", "auscultation of bowel sound at regular intervals (implementation rate 45\%)", and "flushing the pipeline every 4-6 hours (implementation rate 49\%)".

Multivariate logistic regression analysis $(\alpha$ in $=0.05, \alpha$ out $=0.10$ ) was performed. Emergency nurses' knowledge scores of naso-intestinal tube (score $>9$ ), belief (score $>18$ ), and practice (score $>30$ ) were included as dependent variables; and participants' gender, working years, education, level, hospital grade, average daily emergency visit volume were included as factors (See Table 4). The independent variables were shown in Table 5. The results showed that the average daily emergency visit volume, common emergency diseases including circulatory system diseases, and whether the emergency department implements EICU were the influencing factors of emergency nurses' knowledge of naso-intestinal tubes $(\mathrm{P}<0.01)$. Among them, the $\mathrm{P}$-value was 0.41 when the daily emergency visit volume was greater than 500, indicating that the volume of emergency cases greater than 500 was not related to the passing rate of nurses' knowledge of naso-intestinal tube. Hospital grade, education background, and EICU implementation in emergency departments were the influencing factors of emergency nurses' practice in naso-intestinal tube $(\mathrm{P}<0.05)$. Among them, the $\mathrm{P}$-value was 0.176 when the hospital grade was grade II level A, indicating that hospital with grade II level A or below was not correlated with nurses' practice performances on naso-intestinal tube. Gender, education background, and whether the emergency department carries out EICU are the influencing factors of emergency nurses' belief in nasointestinal tube $(\mathrm{P}<0.05)$.

Table 5. Assignment methods of independent variables.

\begin{tabular}{|c|c|}
\hline \multicolumn{2}{|c|}{ Independent variables assignment method } \\
\hline Gender & Male $=1 ;$ Female $=2$ \\
\hline Record of formal schooling & $\begin{array}{c}\text { Technical secondary school }=1 ; \text { College }=2 ; \\
\text { Bachelor }=3 ; \text { Master and higher }=4\end{array}$ \\
\hline Title & $\begin{array}{c}\mathrm{N}=1 ; \text { Nurse practitioner }=2 ; \text { Supervisor nurse }=3 ; \\
\text { Deputy Director nurse and above }=4\end{array}$ \\
\hline Hospital grade & $\begin{aligned} \text { Grade III level A } & =1 ; \text { Grade } \mathrm{III}=2 ; \text { Grade } \mathrm{II} \text { level } \\
\mathrm{A} & =3 ; \text { Grade } \mathrm{II}=4\end{aligned}$ \\
\hline Average emergency visit volume & $0-200=1 ; 200-500=2 ; 500-800=3 ;>800=4$ \\
\hline EICU implementation & $\mathrm{N}=0 ; \mathrm{Y}=1$ \\
\hline Respiratory system diseases & $\mathrm{N}=0 ; \mathrm{Y}=1$ \\
\hline Circulatory system diseases & $\mathrm{N}=0 ; \mathrm{Y}=1$ \\
\hline
\end{tabular}

The survey found that $92.13 \%$ of emergency nurses wanted to participate in enteral nutrition-related trainings. Among the reasons for expecting training, $87.8 \%$ were for their ability improvement, $62.6 \%$ were for the medical needs of emergency patients, $81.7 \%$ were for the establishment of tube feeding, $87.64 \%$ were for the procedures of enteral infusion, and $87.32 \%$ were the for prevention and nursing countermeasures of medical complications.

\section{DISCUSSION}

According to the survey on naso-intestinal tube knowledge of emergency nurses, $22.31 \%$ of nurses "do not know", $50.72 \%$ of nurses "have heard of", $23.11 \%$ of nurses "have used it under instruction", and only $3.85 \%$ of nurses "have systematically trained and used it". The total score of naso-intestinal tube knowledge of nurses was $8.24 \pm 5.02$, and the qualified number was 314 (52.2\%). These results suggest that emergency nurses have limited knowledge of the naso-intestinal tube; and therefore, relevant education and training are needed. 
Table 4 Multiple logistic regression analysis of emergency nurses' knowledge, belief, and practice scores on naso-intestinal tube (n=602, Score, mean \pm SD).

\begin{tabular}{|c|c|c|c|c|c|c|c|c|c|c|}
\hline \multirow{2}{*}{ Items } & \multirow{2}{*}{ Number } & \multicolumn{3}{|c|}{ Knowledge } & \multicolumn{3}{|c|}{ Belief } & \multicolumn{3}{|c|}{ Practice } \\
\hline & & Score & Wald & Pvalue & Score & Wald & P value & Score & Wald & P value \\
\hline \multicolumn{11}{|l|}{ Gender } \\
\hline Male & 109 & $9.14 \pm 4.87$ & 0.136 & 0.713 & $22.75 \pm 6.21$ & 10.457 & 0.001 & $28.66 \pm 16.83$ & 2.449 & 0.118 \\
\hline Female & 493 & $8.04 \pm 5.04$ & . & . & $24.05 \pm 4.70$ & . & . & $30.01 \pm 19.11$ & . & . \\
\hline \multicolumn{11}{|l|}{ Education } \\
\hline Technical secondary school & 28 & $6.82 \pm 4.78$ & 0.775 & 0.379 & $22.79 \pm 6.12$ & 779.613 & .000 & $27.07 \pm 19.74$ & 1313.975 & .000 \\
\hline College & 192 & $6.99 \pm 5.19$ & 0.602 & 0.438 & $23.99 \pm 4.81$ & 2950.021 & .000 & $28.02 \pm 20.48$ & 5255.707 & .000 \\
\hline Bachelor & 378 & $8.93 \pm 4.82$ & 0.215 & 0.643 & $23.79 \pm 5.08$ & . & . & $30.74 \pm 17.69$ & . & . \\
\hline Master or higher & 4 & $12.50 \pm 3.11$ & . & . & $25.25 \pm 1.89$ & . & . & $40.75 \pm 3.30$ & . & . \\
\hline \multicolumn{11}{|l|}{ Hospital grade } \\
\hline Grade III level A & 418 & $8.88 \pm 4.72$ & 6.428 & 0.011 & $23.59 \pm 5.24$ & 1.007 & 0.316 & $31.67 \pm 17.56$ & 8.602 & 0.003 \\
\hline Grade III & 62 & $8.45 \pm 5.28$ & 3.646 & 0.056 & $25.87 \pm 3.45$ & 1.336 & 0.248 & $30.76 \pm 19.10$ & 6.61 & 0.01 \\
\hline Grade II level A & 97 & $6.11 \pm 5.19$ & 0.082 & 0.774 & $23.80 \pm 4.58$ & 0.702 & 0.402 & $23.99 \pm 20.71$ & 1.833 & 0.176 \\
\hline Grade II & 25 & $5.16 \pm 5.48$ & & . & $22.52 \pm 5.28$ & . & . & $17.84 \pm 20.42$ & . & . \\
\hline \multicolumn{11}{|l|}{$\begin{array}{c}\text { Daily emergency visit } \\
\text { volume }\end{array}$} \\
\hline $0-200$ & 131 & $8.20 \pm 4.71$ & 6.481 & 0.011 & $23.41 \pm 6.02$ & 1.407 & 0.235 & $30.42 \pm 18.48$ & 2.731 & 0.098 \\
\hline
\end{tabular}




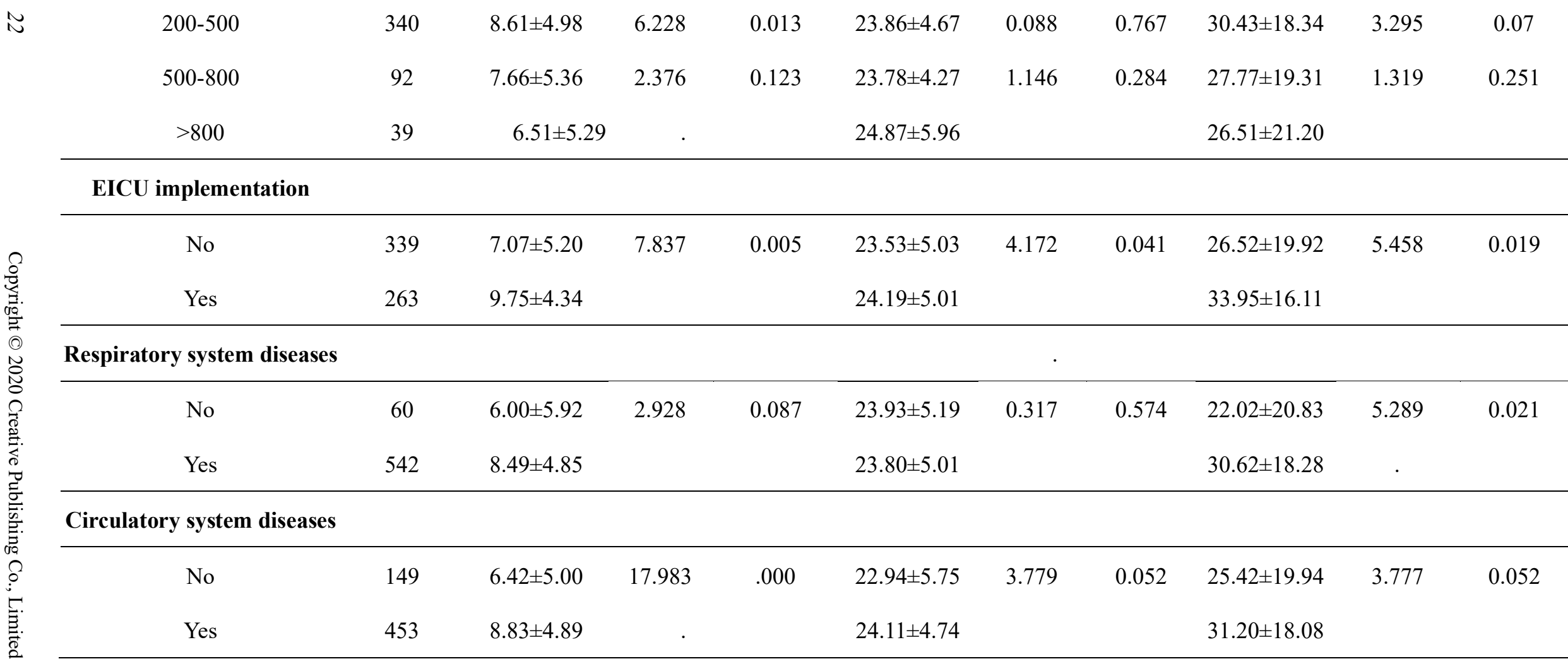


Huang Xuan-jie et al. To investigate and analyze the current using situation of nasointestinal tube in emergency department Medical Research ISSN 2664-0333 eISSN 2664-0341 Volume 2 Issue 2 http://dx.doi.org/10.6913/MRHK.202006_2(2).0003

The results showed that $83.2 \%$ of nurses agreed with the statement "it is very important to master the theoretical knowledge and skills related to naso-intestinal tube". $80.7 \%$ of nurses agreed, "naso-intestinal nutritional support is very helpful for patients with gastric retention, gastric emptying disorders, and high risks of associated pneumonia". $77.2 \%$ of the nurses agreed, "the implementation of naso-intestinal nutritional support protects the interests of patients". $72 \%$ of nurses agreed with the statement that "it makes sense to develop naso-intestinal tube technology in the emergency department". However, nurses' practice performance was not satisfied. Only $42.5 \%$ regularly or consistently "monitoring gastric residuals". $45.7 \%$ of nurses were able to "auscultate patients' bowel sounds regularly or consistently". $49.5 \%$ of the nurses could "flush the pipe every $4 \sim 6 \mathrm{~h}$ " frequently or continuously. It indicates that, at present, although it is easy for nurses to learn the knowledge of naso-intestinal tube, the clinical practice may be a challenge, and the reasons leading to this phenomenon are various. $72.7 \%$ of nurses agreed with the statement "no systematic training", and $52.5 \%$ of nurses in their department have no unified operating guidelines. These might be the influencing factors for the poor implementation and practice of naso-intestinal tube in nursing. Emergency medicine has always been focusing on cardiopulmonary and brain resuscitation, rescue, recovery and maintenance of patients' hemodynamics, and reduction of patients' mortality. The concept of achieving the above goals makes emergency medical staffs pay insufficient attention to the basic care of patients, including naso-intestinal care. Therefore, it also limits the performance of nurses' practice on nasto-intestinal tubes. According to Xinhua He, currently, elderly patients with multi-system diseases account for the majority of the patients in the emergency observation room of most hospitals, especially in the Grade III general hospitals ${ }^{[9]}$. The retention of critically ill and senile patients requires emergency nurses to have not only experienced first aid skills, but also good performances in the basic care of patients, including the nursing and management of various pipelines including naso-intestinal tubes.

Most of the emergency patients are suffering from trauma, coronary cardiovascular disease, stroke and other emergency diseases, and many of the diseases endanger their lives. Nutritional support is an important component of comprehensive treatments for severe patients, and gastro-intestinal nutrition is the first choice ${ }^{[14]}$. Enteral nutrition support is an important measure to maintain the physiological functions of organs, reduce protein consumption, improve immunity and prognosis in critically ill patients, and is also the most basic and important component of comprehensive treatment measures for critically ill patients ${ }^{[11-13]}$. Hyunjung et al. found that the intake of calories and major nutrients of the naso-gastric tube was significantly higher than that of nasogastric tube in the enteral nutrition of critically ill patients ${ }^{[6]}$.

According to the survey by the end of 2016, the number of people aged $\geq 60$ years in China has reached 231 million ${ }^{[4]}$. By 2050, the number of elderly people in China will reach 483 million, accounting for $34.1 \%$ of the whole population. In 2012, the Chinese Medical Association Enteral Nutrition Branch of the national elderly inpatients' nutrition survey results showed that the incidence of malnutrition in elderly patients reached 14.7\%. The nutrition and Health Report of The Elderly population in China in 2015 pointed out that $48.4 \%$ of the elderly population in China had poor nutritional status. In 2012, the total economic burden of malnutrition among the elderly was 84.14 billion yuan, of which 63.93 billion Yuan was directly borne, accounting for $10.6 \%$ of the treatment cost of the elderly population ${ }^{[8]}$, placing a heavy burden on social security and families. The demand and utilization rate of the elderly population for medical and health care in China shows an obvious trend of growth, especially the nutrition problem of elderly patients becomes increasingly prominent. Strengthening clinical nutrition management has become an important part of comprehensive treatment for elderly patients, and it is a problem that needs to be paid attention to and solved in the process of sustainable development of medical institutions ${ }^{[15]}$. 
Huang Xuan-jie et al. To investigate and analyze the current using situation of nasointestinal tube in emergency department Medical Research ISSN 2664-0333 eISSN 2664-0341 Volume 2 Issue 2 http://dx.doi.org/10.6913/MRHK.202006_2(2).0003

Under the new situation, naso-intestinal tubes will be increasingly widely used in the emergency department, but at present, the knowledge of emergency nurses on the naso-intestinal tube is limited, and emergency nurses need to improve their naso-intestinal tube-related nursing skills.

With the increasing aging of the population in China, it is critical for emergency nurses to master the naso-intestinal tube technique. However, this study has several limitations. First, the questionnaire for this survey was designed by the researchers; although the questionnaire was based on relevant literature, its reliability and validity still need to be further verified. Secondly, this study used a convenience sampling method to sample 602 nurses from 126 general hospitals in Guangdong province. It should be noted that this method was not completely randomized; the male to female ratio was quite different in this study, and the representativeness of the sample needs to be further improved. Therefore, it is recommended that future studies should be conducted with larger sample sizes and multicenter randomized sampling surveys to make the results more convincing.

This survey indicates that emergency nurses have good beliefs about patients' naso-intestinal tube; however, their knowledge and practice performances need to be improved. Importantly, manager levels should actively carry out targeted learning and training based on relevant influencing factors analyzed by knowledge, belief, and practice in this study. Meanwhile, the emergency department should also actively develop clinical nursing practice guidelines and norms of the naso-intestinal tube to improve the knowledge level and clinical practice of emergency nurses, as well as to promote the application of naso-intestinal tube technique in the emergency clinic.

\section{ACKNOWLEDGEMENT}

The research was performed in Emergency Department, the Seventh Affiliated Hospital, Sun Yat-sen University.

\section{STATEMENT}

There is no conflict of interest in this article.

\section{REFERENCES}

[1] Wang Xiaoling, Jiang Xuemei, Dai Yao. Research Progress in Application and Nrsing of Nasoenteric Tube [J]. Chinese Journal of Nursing, 2014, 49 (12): 1506-1510.

[2] Lin Lizhen, Zhuang Caijuan, Zhang Hui, Ming Jianqing, Fang Lu Jin. Investigation and Analysis of Nurses'Knowledge about Nasoenteric Tube [J]. General Practice, 2018, 16 (16): 2010-2013.

[3] Cha Liling, Zhou Song, Wang Jianning. Investigation of Early Activity Cognition and Behavior of ICU Nurses [J]. Chinese Journal of Nursing, 2018, 53 (02): 221-225

[4] Hu Wen. 2017 Expert Consensus on Home Nutrition Management for Elderly Patients. Chinese Journal of Evidence-Based Medicine 2017, 11 (17): 1251-1259.

[5] Ma Yuxiu, Liu Zhiying, Wang Xia. Comparison of adverse reactions between duodenogastric tube and spiral nasoenteric tube in elderly patients [J]. Journal of Nursing Science, 2009, 16 (3A): 58-59.

[6] Hyunjung K, Nancy AS, Erika SF, et al. Why patients in critical care do not receive adequate enteral nutrition? A review of the literature [J]. Journal of Critical Care, 2012, 27:702-713. 
Huang Xuan-jie et al. To investigate and analyze the current using situation of nasointestinal tube in emergency department Medical Research ISSN 2664-0333 eISSN 2664-0341 Volume 2 Issue 2 http://dx.doi.org/10.6913/MRHK.202006_2(2).0003

[7] Stephen AM, Robert GM, Vincent WV, et al Guidelines for the Provision and assessment of nutrition support therapy in the adult critically ill patient: Society of Critical Care Medicine (SCCM) and American Society for Parenteral and Enteral Nutrition (A.S.P.E.N.) [Fig. J]. Journal of Parenteral and Enteral Nutrition, 2009, 33 (3): 277-317.

[8] Chai Peipei, Zhang Yuhui, Wanquan, et al. Study on the economic burden of malnutrition in the elderly in China. China Health Economics, 2016, 35 (3): 13-16.

[9] He Xinhua, Gaoli, Tengfei, et al. A new charging scheme in emergency department observation unit under Binjing's basic medical insurance [J]. Chin Med J, 2014, 127 (18): 3286-3290.

[10] Guo Lili, Chen Gang, He Xinhua. Nutritional status analysis of elderly patients in emergency department [J]. Chinese Medical Records, 2018, 19 (9): 84-88.

[11] Li Xiuchuan, Chen Lianfang. Observation on the effect of bedside blind nasoenteric tube insertion in nutrition of critically ill patients [J]. Journal of Bengbu Medical College, 2016, 41 (6): 825 - 829.

[12] Substitutes, Zhang Shuqin, Zhang Zhaoyuan, etc. Effect of Electroacupuncture Stimulation on Success Rate of Blind Insertion Spiral Nasoenteric Tube in Critically Ill Patients [J]. Chinese Journal of Emergency Medicine, 2015, 24 (11): 2009-2011.

[13] Sun Jinjie, Xu Xujuan. Progress in Nutrition [J]. World Chinese Journal of Gastroenterology, 2014, 22 (11): $1525-1530$.

[14] Xu Can, Wang Wenli, Yi Rongfang. Effect of blind insertion of spiral nasoenteric tube by intermittent advancement in neurosurgical critically ill patients [J]. Chinese Journal of Nursing, 2015, 50 (4): 439-441.

[15] Ding Yaming, Yu Ting. Nutritional Status of Elderly Patients Seeking Medical Attention [J]. Chinese Journal of Gerontology, 2017, (18) :4660-4661.DOI:10.3969/j.issn .1005-9202.2017.18.108. 